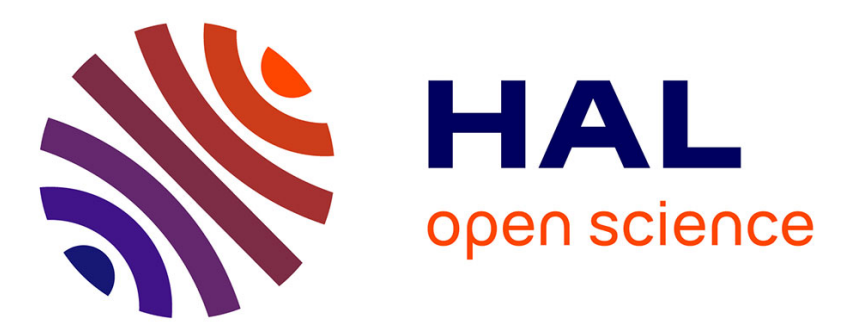

\title{
Isometric Unfolding of Stratigraphic Grid Units for Accurate Property Populating: Mathematical Concepts
}

Sébastien Horna, Chakib Bennis, Houman Borouchaki, Christophe Delage, Jean Francois Rainaud

\section{To cite this version:}

Sébastien Horna, Chakib Bennis, Houman Borouchaki, Christophe Delage, Jean Francois Rainaud. Isometric Unfolding of Stratigraphic Grid Units for Accurate Property Populating: Mathematical Concepts. European Conference on the Mathematics of Oil Recovery, Sep 2010, Oxford, United Kingdom. hal-00991385

\section{HAL Id: hal-00991385 \\ https://hal.science/hal-00991385}

Submitted on 15 May 2014

HAL is a multi-disciplinary open access archive for the deposit and dissemination of scientific research documents, whether they are published or not. The documents may come from teaching and research institutions in France or abroad, or from public or private research centers.
L'archive ouverte pluridisciplinaire HAL, est destinée au dépôt et à la diffusion de documents scientifiques de niveau recherche, publiés ou non, émanant des établissements d'enseignement et de recherche français ou étrangers, des laboratoires publics ou privés. 


\title{
Isometric Unfolding of Stratigraphic Grid Units for Accurate Property Populating: Mathematical Concepts
}

\author{
S. Horna ${ }^{1}$, C. Bennis ${ }^{1}$, H. Bourouchaki ${ }^{2}$, C. Delage ${ }^{1}$, J. F. Rainaud ${ }^{1}$ \\ ${ }^{1}$ IFP Energies Nouvelles, ${ }^{2}$ GAMMA3 - INRIA
}

\begin{abstract}
In traditional methods used to populate stratigraphic units, the distortions can be very important and affect the setting up of the static and dynamic parameters necessary to the reservoir simulation, hence the simulation results. These distortions result from the mapping between the original curvilinear stratigraphic grid and the intermediate cartesian grid in which the property populating is processed.
\end{abstract}

To minimize the deformation and improve the populating process, we propose a new original isometric unfolding process based on the minimization of the elastic tensor deformation. This method could be applied for every type of deposit: horizontal, parallel to top, parallel to bottom, proportional.

Starting from a structural model defined into a coordinate line grid, the user chooses a reference iso-chronological level represented by a triangulated surface. This level can be the top, the bottom or any other characteristic surface of a litho stratigraphic unit. The contacts between this surface and fault surfaces are explicitly extracted as coincident edges. These coincident edges are used to constraint an unfolding process which minimizes the elastic deformation tensor on the whole surface and then, respecting the above constraints, join the fault lips opened by geological tectonic events.

To exploit this first 2D Unfolding for volumetric purposes, we use the coordinate line grids as a $3 \mathrm{D}$ support and define a complete mapping between the original curvilinear structural grid and its image in the deposition (unfolded) domain. This mapping which expresses the geometrical transformation between the today's world and the depositional period could now be used to transport today's well trajectory stations in the unfolded volume for geostatistical computation and, on the way back, define the geometrical relations needed for upscaling.

In this paper we will focus on mathematical concepts of the algorithms used in the whole unfolding process and present some results throw actual case studies.

\section{Main Objectives:}

Present the mathematical concept and methodology for a new unfolding method based on the minimization of the elastic tensor deformation. With this method, we will be able to associate to a stratigraphic grid unit in structural position to its cell to cell image in the depositional space. This mapping could be used to transport well trajectory stations in the unfolded volume for geostatistical computation.

\section{New aspects covered :}

We propose a new original isometric unfolding process based on the minimization of the elastic tensor deformation. We apply this algorithm to unfold iso-chronological surfaces and to unfold volumes in several depositional modes: Top and bottom conformable, proportional and parallel to any surface. 


\section{Introduction}

The method presented in this article concerns the domain of the oil exploration, and more particularly an important phase of the characterization of the oil reservoirs: populate a lithostratigraphic unit (represented by a fine stratigraphic grid, figure 1) by the rock properties in the center of the cells of this grid (facies, porosity, permeability).

In many cases geostatistic methods are used to spread the information measured along the drilling trajectories on the totality of the fine stratigraphic grid. These geostatistic methods deliver more reliable and faster results when they are processed on a 3D regular cartesian grid representing the volume of sediment accumulated during the deposit period.

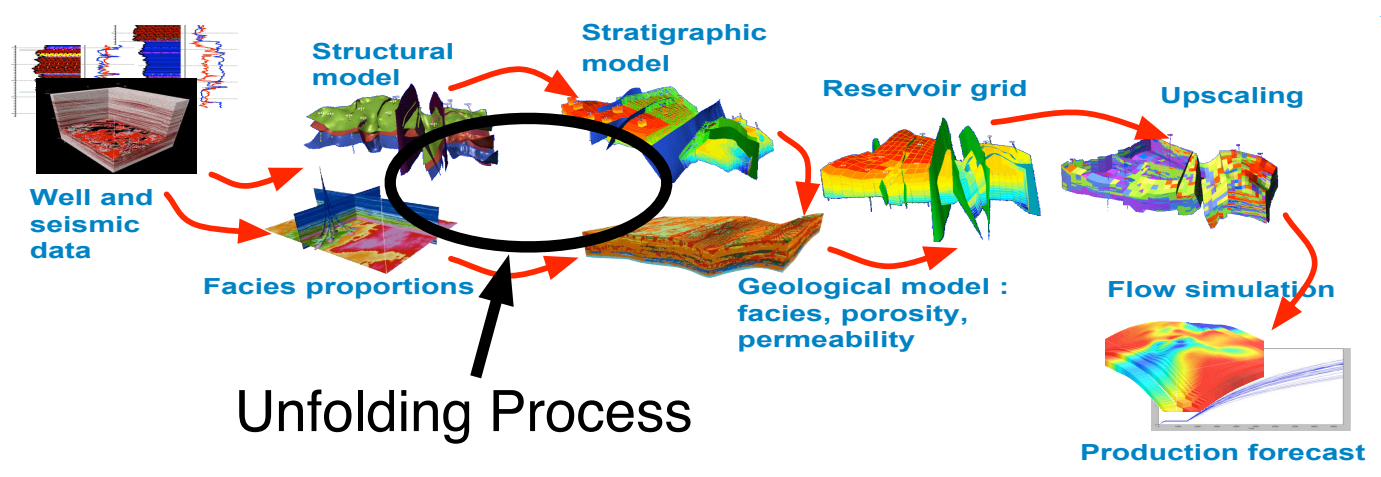

Figure 1 Reservoir characterization workflow.

Then, to realize geostatistic computing, our strategy consists in using the cartesian metrics of the deposit space to realize the simulations geostatistic. For this strategy, it is necessary to allocate to every point of the geographical space a position in the deposit space (see figure 2). This means restoring all lithostratigraphic units of the reservoir, that is crossing towards the past its tectonic history of fault sets.

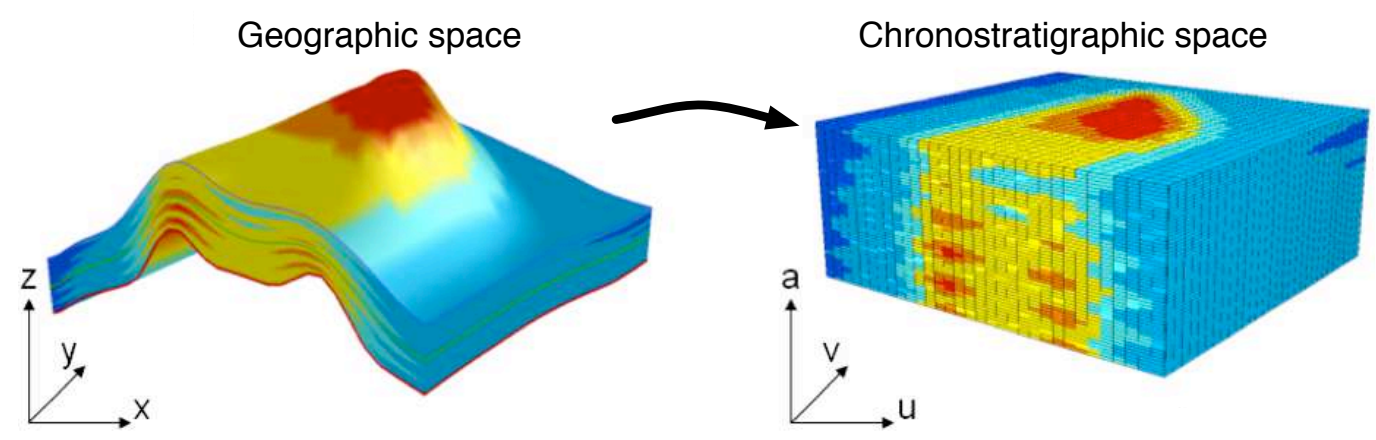

Figure 2 Data set and result of unfolding method: (a) stratigraphic grid (b) deposit space.

Our method will provide a way to process independently one or more lithostratigraphic unit and facilitate partial update. In fact, with the wide usage of geo-modelling tools, users could have the need to enhance their previous geostatiscal population without rebuilding an entire stratigraphic model. This may be useful if a new well delivers updated data. In this case, if the user would like to apply new geostatiscal population methods or would like to constraint the property population after some non consistent fluid flow simulation tests. For this opportunity, they often need to extract with a best precision a single lithostratigraphic unit and "repopulate" it with other accurate methods. 
In previous works, a very often used classic method consists in building a flat regular grid in the deposit domain. One layer limit is chosen as the reference level on which the sediments were deposed. This layer limit is horizontally flattened. The cells in $\mathrm{X}, \mathrm{Y}, \mathrm{Z}$ directions are then replaced relatively to this reference level in a regular cartesian grid. Dimensions I, J, and $\mathrm{K}$ were calculated from the original lithostratigraphic unit dimension. Properties measured in a $[\mathrm{i}, \mathrm{j}, \mathrm{k}]$ original cells transported in the same $[i, j, k]$ cell of the regular stratigraphic grid defined (see figure 3.b).

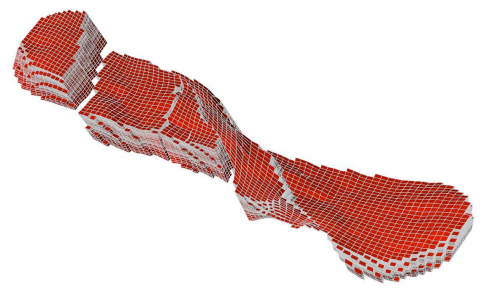

(a)

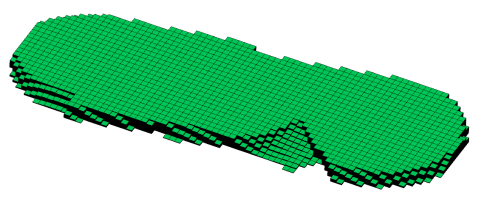

(b)

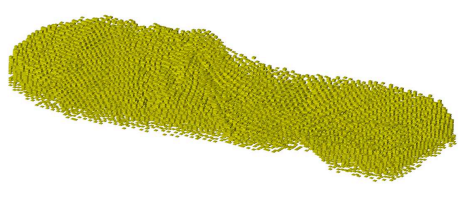

(c)

Figure 3 Deposit space comparison (a) stratigraphic folded grid (b) deposit space represented by a regular grid (c) deposit space computed by our unfolding process.

Unfortunately, using a regular grid produce important geometric deformation. To reduce this deformation, we propose a new unfolding process based on a isometric surface unfolding. In this process, we can start from the same layer limit than the classical method, and use it as a reference surface on which the sediments are deposed. But after having flattened this unfolded reference all layers included between top and base are straightened. The deformation applied on each reference surface point is computed along the corresponded coordinate-line in the stratigraphic grid. The process allow to minimize the grid deformation (see figure 3.c).

\section{Method description}

From the isometric unfolding of a selected Reference Surface (named RS there after), all interlayer limits between top and bottom layers selected by the user will be straightened. For that purpose, RS must be extracted first of all then unfolded. Secondly, all surfaces selected by the user are unfolded according to RS.

This work is composed of three steps (see figure 4):

1. extraction and triangulation of a reference surface;

2. isometric unfolding of the reference surface ;

3. deformation of the wholes lithostratigraphic volume in function of the RS deformation realized during its isometric unfolding. 


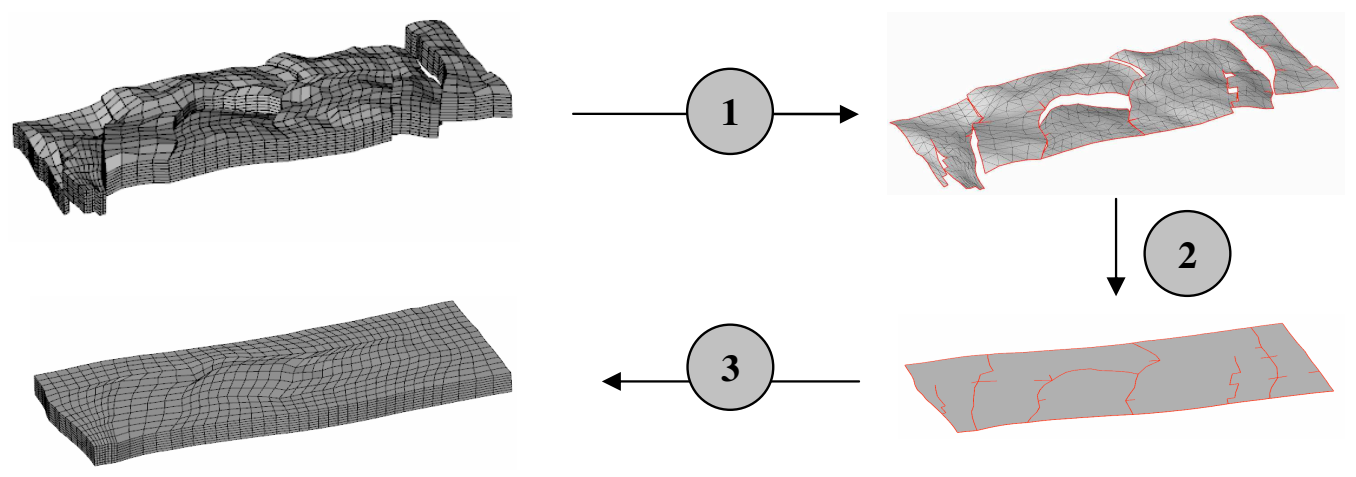

Figure 4 Unfolding workflow representation: (1) reference surface extraction (2) reference surface unfolding (3) deposit space computing.

\section{Stratigraphic unit construction}

Inside a reservoir grid, stratigraphic unit are represented by a set of adjacent layers. Starting from this set of layer, we build a stratigraphic grid, which represent a discret subterranean formation, and its structure (layout organization of the sedimentary layers, faults, etc.). This stratigraphic grid is defined in an orthonormal coordinate system (x, y, z). So, every grid cells are located, at the current instant, by geographical address and coordinates $\mathrm{x}, \mathrm{y}$ and $\mathrm{z}$ (depths). The stratigraphic unit is bounded by a upper surface and a lower surface and represents a homogeneous sedimentary layer (see figure 5).

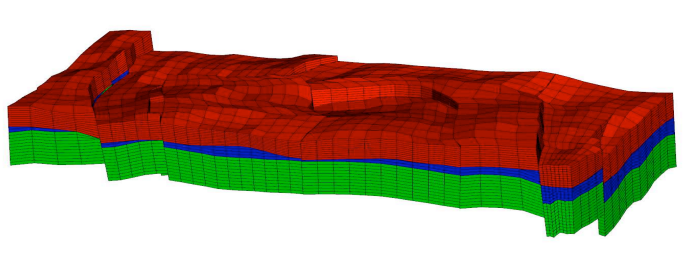

(a)

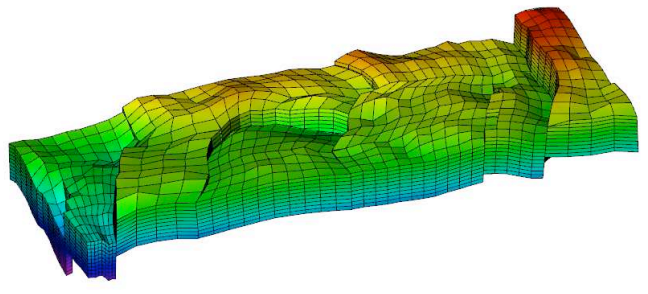

(b)

Figure 5 Stratigraphic unit construction (a) unit detection (b) unit construction from layer 0 to 7.

\section{Extraction and triangulation of the reference surface}

To operate, the user isolates from a $I, J, K$ coordinate-line grid a stratigraphic unit between two $K$-values ( $K-$ top and $K$-bottom). In this unit, the user choose an inter layer limit as reference surface between $K$-top and $K$-bottom. This layer is being represented by all grid points having the same $K$-value.

All faces included in this layer are extracted from the grid to produce the RS. This surface is triangulated in order to be used by the unfolding algorithms and the horizon / fault contact sealing. The preprocessing of RS extraction from a stratigraphic pillar grid $(I, J, K)$ is made in two steps:

1. a matrix of face is defined from the K-layer and a direction (top or bottom - i. e. topper or lower faces). It contains the surface points of the triangulated surface;

2. topology is checked and corrected where mistakes are detected. 


\section{Construction of faces matrix}

This matrix contains every face of each layer cell, for the given direction. The aim is that all matrix faces will contain an index on its four incident vertices. So, the top-face (resp. bottom) of a unit cell of grid contains 4 indications of vertices numbered in order logic (see figure 6.b).

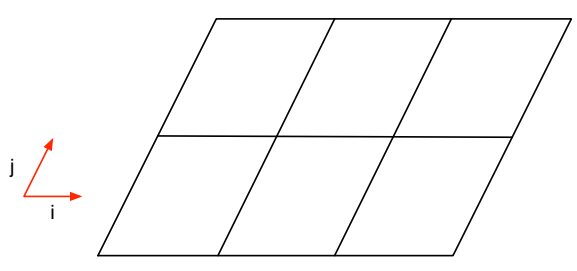

(a)

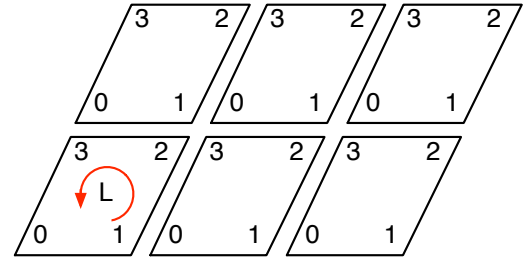

(b)

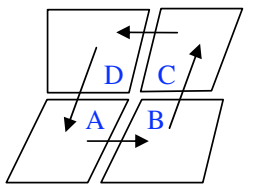

(c)

Figure 6 Surface vertices construction from grid vertices. Each face is incident to 4 vertices.

Surface vertices are generated by a iteration on of all incident nodes of the $K$-layer cells. All vertices of every node are covered in a circular way $(A \rightarrow B \rightarrow C \rightarrow D$, figure 6.c). For each vertex, its curvilinear coordinate is compared with the previous vertex. If this one is different, a new vertex is added to the generated surface.

Note : with this process, if all node vertices have the same coordinates, those vertices correspond to the same unique vertex in the generated surface.

\section{Topology checking and fault lips association}

To use our unfolding process, we need manifold surfaces. To generate manifold surfaces, a specific process is applied on nodes. The correction must be made on faces matrix, before the generation of triangulated surface to guarantee a correct fault detection. For that purpose, the topology is corrected node by node "by cutting" the grid (i.e. nodes are duplicated to eliminate the presence of non manifold area).

To keep the explicit information contained in the stratigraphic grid, for every cut node, a reference to the corresponding node is stored (see figure 6.b). This data will allow to put in correspondence the fault lips during unfolding process (see figure 6.c).

\section{Triangulation and topological links set up on RS}

To be as generic as possible, the flattering process was developed on triangulated surfaces associated by topological links. In this paper, as we are starting from $I, J, K$ coordinate 3D grids (see figure 7.a), we need to generate the triangulated surfaces from $2 \mathrm{D}$ grids extracted from $3 \mathrm{D}$ grids and to associate equivalent horizon / faults contacts.

A first step consists in extracting preexisting fault planes (see figure 7.b) along with we will be able to associate correspondent edges (edges of horizon / faults contacts), which edges were located at the same place during the deposit period and moved by tectonic events. 


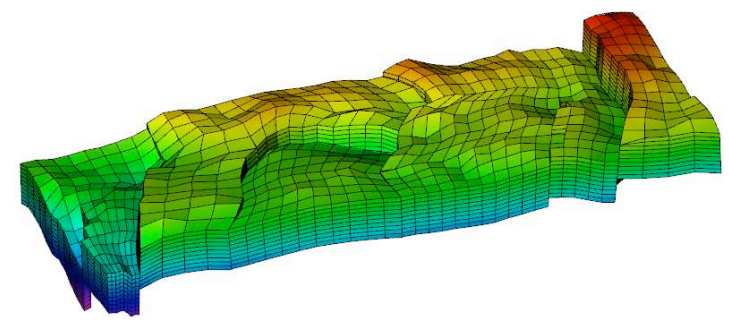

(a)

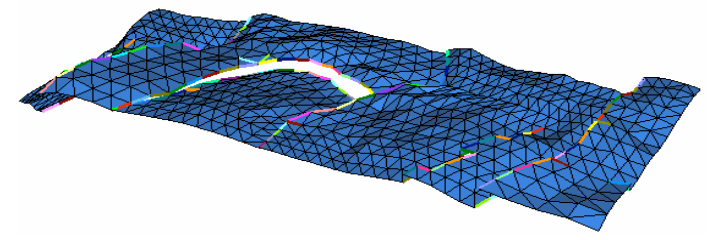

(c)

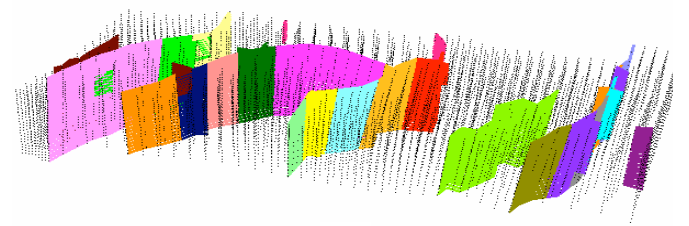

(b)

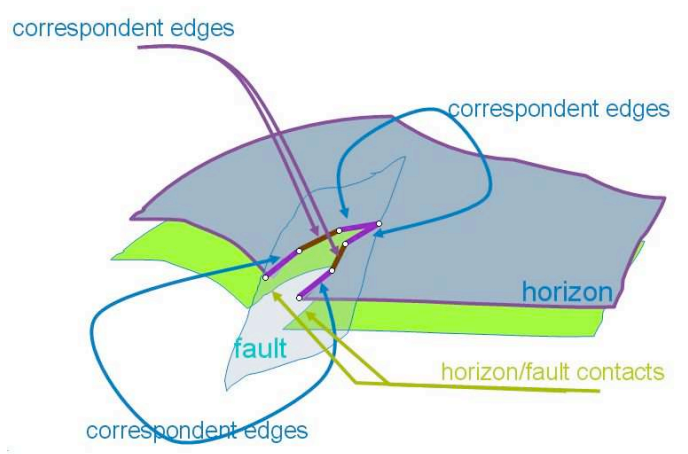

(d)

Figure 7 Explicit information extraction (a) stratigraphic unit (b) fault detected in stratigraphic grid (c) surface extracted with fault lips correspondence stored (d) fault contacts and correspondent edges.

Then, the triangulation of the different patchs (see figure 7.c) representing RS are cleaned (sometimes, the original coordinate line grid is not explicit enough). At least, using this triangulation as the base of the geometry, all horizon / faults contacts are explicitly tagged and all correspondent edges are topologically linked from one side to the other side of the horizon along all horizon / fault contacts. This preprocessing is mandatory before unfolding (see figure 7.d).

\section{Isometric unfolding of reference surface}

The first step of the unfolding process is applied on the RS. This process is computed by using a specific extension of Aplat, software developed by university of technology of Troyes [1].The original Aplat process is used to flatten triangulated surface without tears. A specific code was developed here to associate horizon fault contacts separated during tectonic periods.

Isometric unfolding works in the following way: from a 3D folded surface, we compute an unfolded plane surface, in which the deformations are minimal. The criterion chosen is a minimization of the deformation of tensor elastic deformation. During the deformation, all " $Z$ " coordinate are computed to obtain a plane surface. At the level of the faults, both horizon / fault contacts are put in correspondences and smoothed "to block" holes at the level this one. Figure 8 illustrates the result of the isometric unfolding of a surface. 


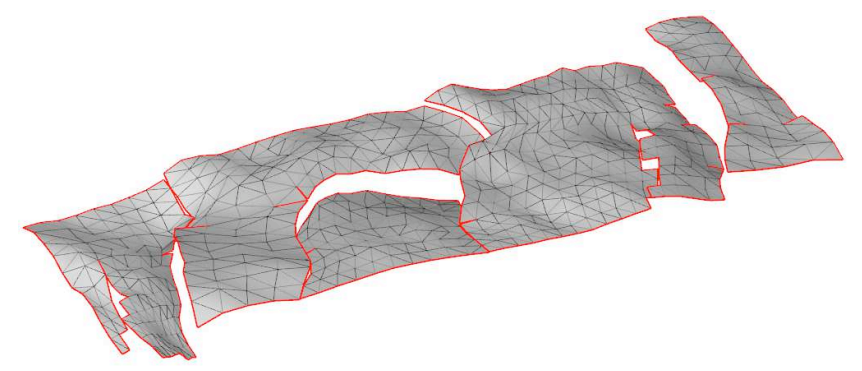

(a)

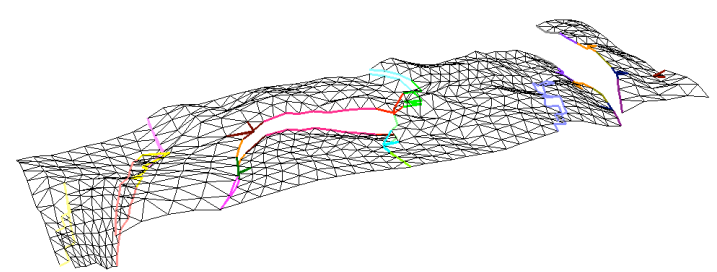

(b)

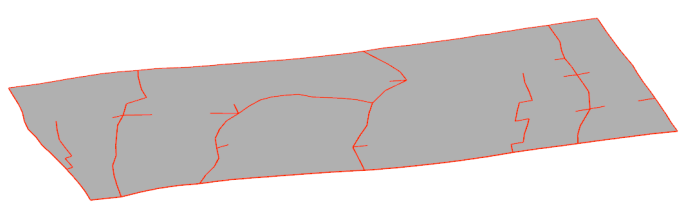

(c)

Figure 8 Isometric unfolding: (a) folded 3D surface (b) horizon / fault contact correspondence (c) corresponding unfolded surface with fault resticking.

\section{Unfolding of stratigraphic grid}

Then, we need to unfold all layers included in stratigraphic unit. From the unfolded reference surface chosen by user, layers included between bottom and top selected by user are "straightened". For every RS points, a deformation is calculated and applied along all the corresponding coord-lines in the stratigraphic grid (see figure 9).

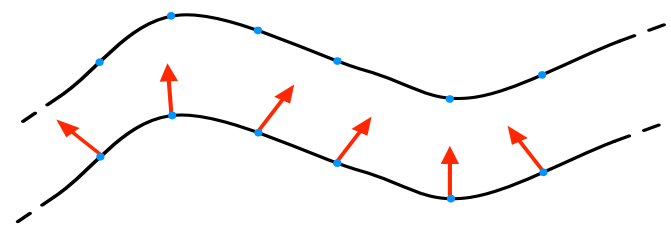

(a)

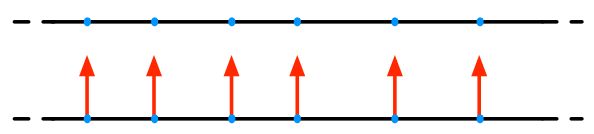

(b)

Figure 9 Surface unfolding by normal straightened (a) folded surface (b) unfolded surface.

This method implies the correspondence between the reference surface and each of the grid coord-line. It is thus necessary to assure that every cood-line corresponds strictly to a point of the reference surface. For it: i) the horizon / fault contacts must correctly coincide (i. e. no hole) ii) the removal of edge to simplify triangular mesh does not have to remove needed information.

The algorithm takes place according to various steps developed afterward:

- correspondence between the points of the triangulated reference surface and the grid nodes;

- for each reference surface point $P$ :

- computing of the transformation T on P (folded surface $\rightarrow$ unfolded surface);

- on all coord-line point crossing $\mathrm{P}$, the transformation $\mathrm{T}$ is applied. 


\section{Correspondance between grid and surface}

To apply the same deformation realized on RS during isometric unfolding to every grid nodes, a correspondence between RS points and grid points must be defined. Every RS vertex is then associated to one coord-line.

Along faults, several vertices can belong to the same coord-line (see figure 10). Each of these vertices must be processed separately even it they belong to the same coord-line. This feature implies a dedicated processing along faults. This will be explain in the following section.

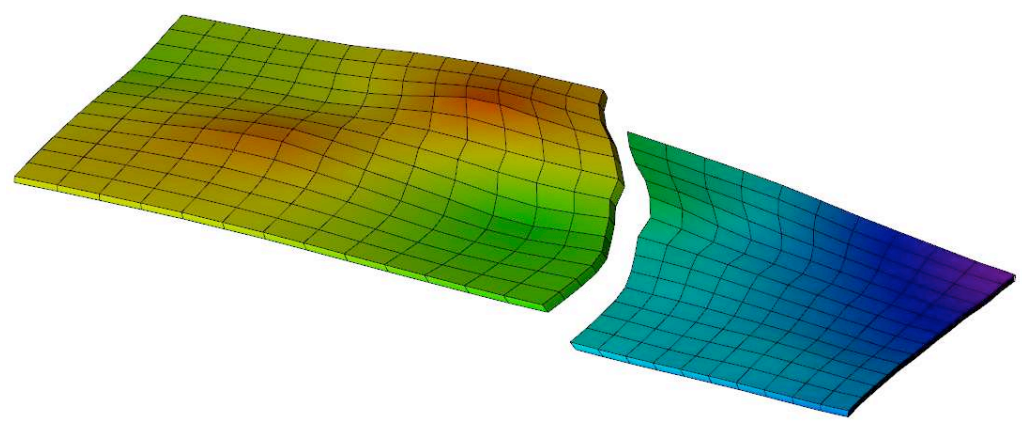

Figure 10 Surface discontinuity representation.

\section{Transformation on each surface point}

To handle all vertices built during the RS unfolding, the corresponding surface coord-lines is processed iteratively (and not on the all grid nodes). For every RS vertices, two cartesian coordinate bases are defined. The one with regard to the unfolded surface (see figure 11), the other one with regard to the folded surface (see figure 12).

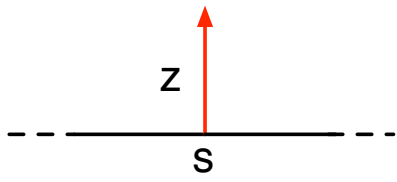

(a)

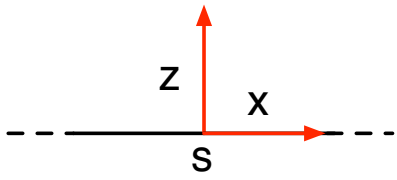

(b)

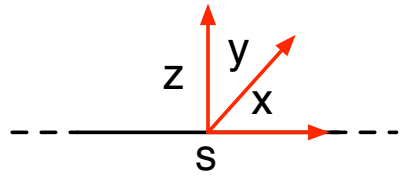

(c)

Figure 11 Cartesian coordinate on vertex $S$ included in flat surface $(a) Z$ is vector $(0,0,1)$, the normal on $S$; (b) $X$ is vector along an incident edge (c) $Y$ is cross product between $Z$ and $X$.

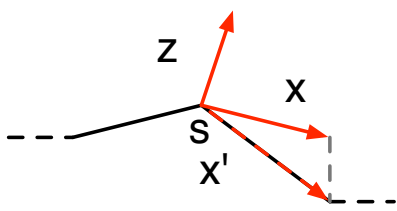

(a)

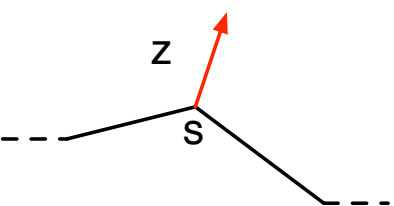

(b)

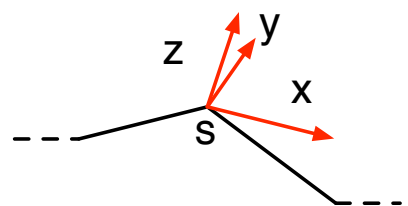

(c)

Figure 12 Cartesian coordinate on vertex $S$ included in folded surface (a) $Z$ is the normal on $S$ (average of each normal incident faces) ; $(b) X^{\prime}$ is included to $Z$ plan, along an incident edge, $X(1,0,0)$ was perpendicular to $Z$; (c) $Y$ is cross product between $Z$ and $X$. 
From produced cartesian coordinate, a transformation by bases change allows to deduct its new position for each horizon. The figure 13 illustrates the unfolding of 3 horizons. In this example, RS is the lower surface 0 , and horizons 0 to 2 are unfolded.

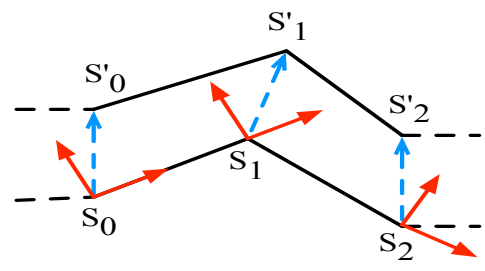

(a)

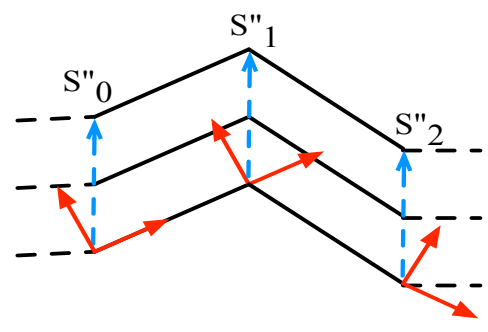

(c)

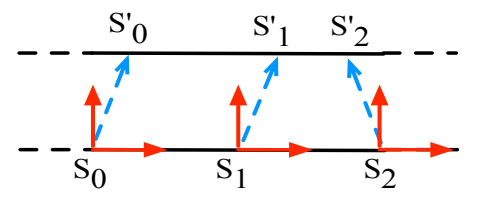

(b)

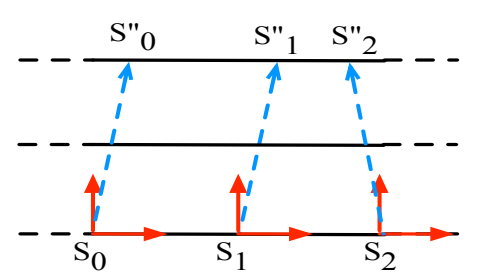

(d)

Figure 13 Unfolding horizons from one reference surface.

But this method, along faults, can produce surface discontinuity. Several RS vertices will correspond to the same (i, j) grid node which referred the same coord-line. During unfolding surface process, horizons / fault contacts will be adjusted to remain in correspondence (see figure 14).

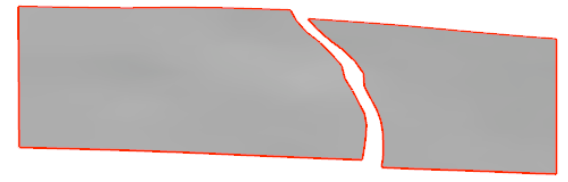

(a)

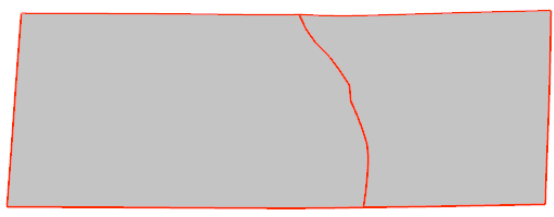

(b)

Figure 14 During unfolding, fault lips are put in correspondence to produce a surface without discontinuity.

In fact, when we are in a non faulted area (see figure 13), we can base the computation of a normal on a vertex on several faces orientations. The normal obtained is reliable. But, when the vertex is located on an horizons / fault contact, we can base this computation on less faces orientations by considering only the faces located in the same horizon patch (on the same side of the fault, figure 15).

It is the reason why a new normal is calculated for every couple of vertices on fault edge, such as $\mathrm{Z}$ is the average of $Z_{1}$ and $Z_{2}$. Normal used in $S_{1}$ and $S_{2}$ being equal, the applied transformations are equivalent (see figure 16).

So, resulting points $S_{1}^{\prime}$ and $S_{2}^{\prime}$ have the same coordinate X, Y. We suggest afterward to the user to keep this result (see figure 16.b), or to compute a new point calculated by interpolation of $S_{1}^{\prime}$ and $S_{2}^{\prime}$ (see figure 16.c). 


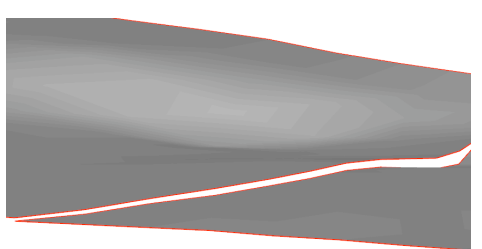

(a)

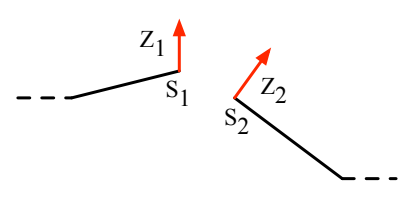

(b)

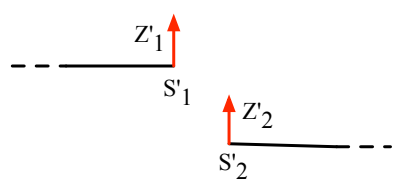

(c)

Figure 15 Discontinuity on unfolded surfaces: (a) bad result produce by a fault unfolding (b) vertices $S_{1}$ and $S_{2}$ correspond to each fault lips, their normal are different; (c) produces vertices $S_{1}^{\prime}$ and $S_{2}^{\prime}$ are disconnected.

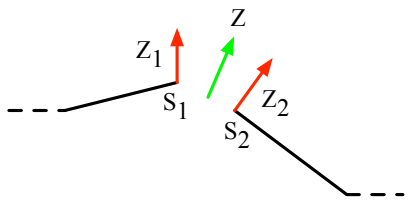

(a)

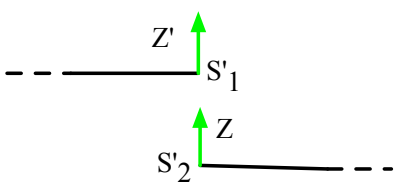

(b)

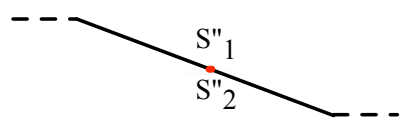

(c)

Figure 16 Discontinuity resolution: (a) $Z$ is an average between normals $Z_{1}^{\prime}$ and $Z_{2}^{\prime}$; (b) solution 1 : resulting vertices $S_{1}^{\prime}$ and $S_{2}^{\prime}$ are computed from $Z(c)$ solution 2 : vertices $S^{\prime \prime}{ }_{1}$ and $S^{\prime \prime}{ }_{2}$ are computed with an interpolation between $S_{1}^{\prime}$ and $S_{2}^{\prime}$.

\section{Unfolding along a coord-line}

Every coord-line can be identified by an address $\mathrm{i}, \mathrm{j}$ corresponding to the address of the origin grid node. So, every RS vertex is associated with one coord-line, from which cartesian coordinate are calculated. The unfolding is so realized along the coord-line for each contained vertices.

\section{Results and comparison}

Figure 17 present the deposit space computed with our unfolding process. To compare grid deformations between classical methods (described in section ) and our unfolding process, we propose to use an approximative representation which objective is to illustrate why the isometric method restore better the distances. Distances separating reference points presented in figure 18 are measured and compared in table 1 .

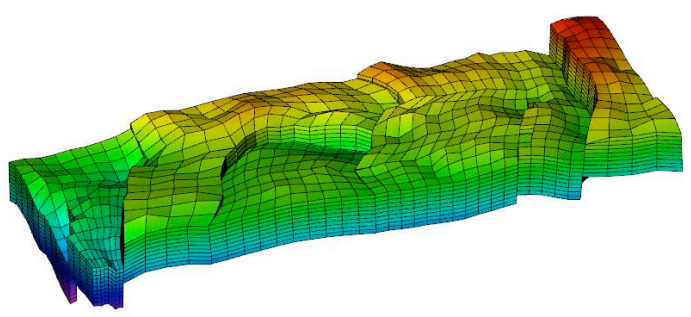

(a)

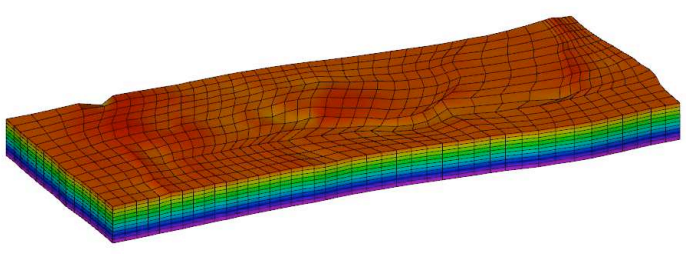

(b)

Figure 17 Results illustration (a) stratigraphic grid (b) resulting deposit space 


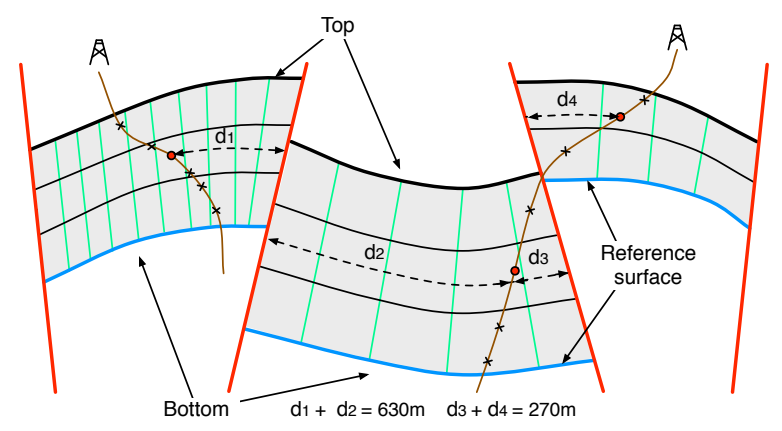

(a)

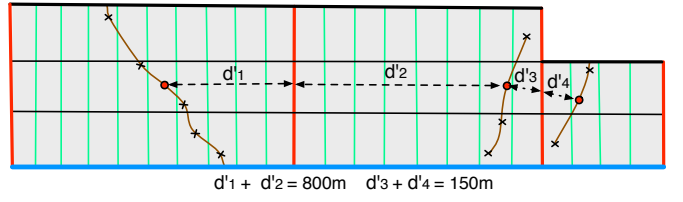

(b)

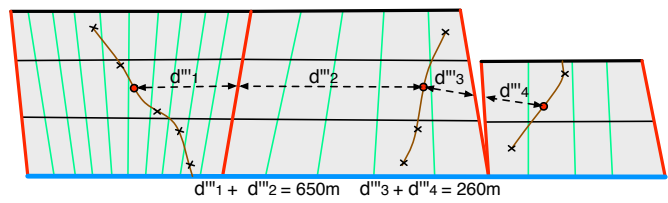

(c)

Figure 18 Representation of deformation (a) original grid (b) deposit space represented by a regular cartesian grid (c) deposit space computed by our isometric unfolding process.

Classic method: the number of cells is respected and the mean value of width of a cell is equal to the average of original cells size. Its illustrated in figure 18.b. We obtain distances $D_{1}=d_{1}^{\prime}+d_{2}^{\prime}$ and $D_{2}=d_{3}^{\prime}+d_{4}^{\prime}$.

Isometric method: the positions of cell corners are computed from the reference surface isometric unfolding. The sizes of cells are not identical to the original cells. In figure 18.c. We obtain distances $D_{1}=d_{1}^{\prime \prime \prime}+d_{2}^{\prime \prime \prime}$ and $D_{2}=d_{3}^{\prime \prime \prime}+d_{4}^{\prime \prime \prime}$. Comparative table of the results

\begin{tabular}{|c|c|c|c|c|}
\hline & $D_{1}$ & variation & $D_{2}$ & variation \\
\hline origin grid & 630 & $0 \%$ & 270 & $0 \%$ \\
\hline classic & 800 & $27 \%$ & 150 & $55 \%$ \\
\hline isometric & 650 & $3 \%$ & 260 & $3,2 \%$ \\
\hline
\end{tabular}

Table 1

In real case, the same effects are felt, what shows the importance of its use to be able to analyze correctly variogram horizons and propose estimations more reliable in geostatistic.

The advantage of our method could ever be more evident if the area organization of the reservoir grid (which most be optimized for fluid flow simulation) looks like the given example presented in figure 19. This is due to the fact that the reservoir grid and the geostatistical grid support can be designed on a total independence. 


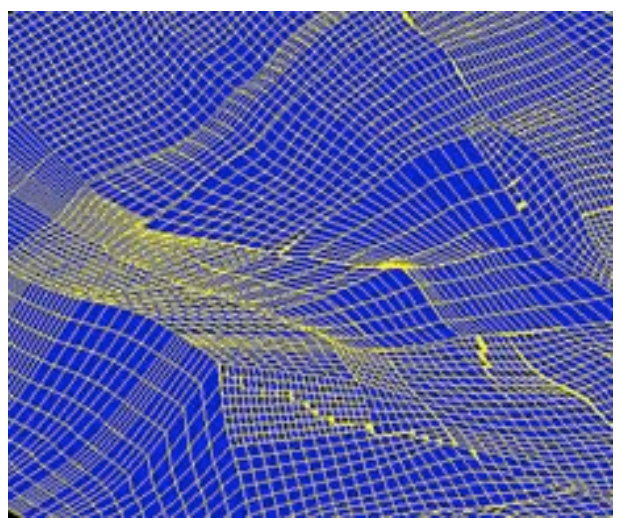

Figure 19 Extraction of the top view of a reservoir grid in a complex faulting geometry.

\section{Conclusion}

The objective to apply our methods is to avoid lateral deformation due to the influence of the setting up of complex fault network as presented in [2] and [3]. Then, the original distance between geological bodies will be restored as it was historically and the computed geostatistical parameters will be more consistent.

Applying this method, as we have seen in the table 1 the user will obtain a more accurate measurement to define vertical and horizontal variograms which are one of the main tools used for geostistical simulations. The quality of the the realization will be a lot improved and no more anisotropy bias will be inherited from reservoir fluid flow gridding constraints. The grid which will be constituted for the geostatiscal population (or any other style) could be totally independently constrained by only geological behaviors.

This method allows to update only a part of the geological model (only one stratigraphic unit) without recomputing all the properties (geometric or petrophysic) of the stratigraphic grid even if we choose a new depositional mode. The ability to process independently the stratigraphic units open the way to update a complete model in parallel on several processors. This will facilitate efficient history matching procedures.

\section{Reference}

[1] Aplat software, developed at University of Troyes.

[2] Galera, C. Bennis, C, Moretti, I., Mallet, J.L. 2003. Construction of coherent 3D geological blocks. Computer and Geosciences, 29, pp 971-984

[3] Gibbs, A. 1983. Balanced cross section from seismic sections in area of extensional tectonics. Journal of Structural Geology 5 (2), pp 153-160. 